sites of aluminium-transition metal quasicrystals, in order to separate the contributions to the diffraction pattern from the sublattices, Janot et al. (Laue-Langevin Institute, Grenoble) also reported the existence of strong diffuse scattering in between the Bragg peaks. Diffuse scattering and peak broadening obviously need to be studied in detail before they can be tentatively correlated with local atomic order or defects such as phasons or wrong connections. These parameters also compromise the significance of the reconstructed Patterson function in periodic sixdimensional space, analysis of which, according to D. Gratias (Centre d'Etudes de Chimie Métallurgique, Vitry), already suffers intrinsically from a degree of freedom on the phases and therefore can only lead to a class of locally isomorphic structures.

From the current state of knowledge

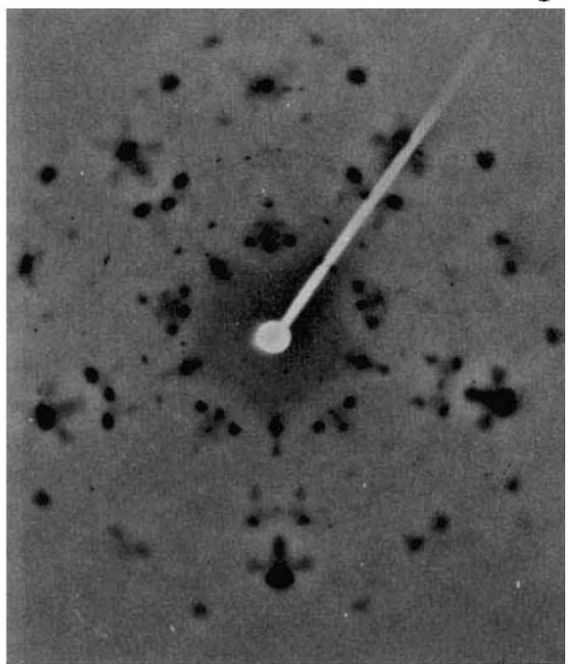

Fig. 2 Laue X-ray diffraction pattern of an $\mathrm{Al}_{6} \mathrm{Li}_{3} \mathrm{Cu}$ quasicrystal; courtesy of Professor F. Dénoyer

about the atomic structure, physical properties of real quasicrystals have been predicted to lie between those of crystals and metallic glasses. Indeed, this trend seems to be observed within the limits of welldefined specimens. For a 'macroscopic' disorder, the concentration gradients, multi-phase states and various types of interface that exist in melt-spun materials (J.L. Verger-Gaugry et al., Lab. de Thermodynamique et Physico-Chimie Métallurgiques, Grenoble) may be the cause of contradictions between some recently reported measurements.

J.B. Suck (Kernforschungszentrum, Karlsruhe), using inelastic neutron scattering, found that the atomic dynamics of icosahedral $\mathrm{Pd}_{58.8} \mathrm{Si}_{20.6} \mathrm{U}_{20.6}$ are very similar to those of the metallic glass of the same composition, both having a generalized vibrational density of states different from that of the fully crystallized state. Quasicrystals and glass share low-energy modes, with the same dependence on momentum transfer. The vibrational part of the specific heat calculated from the density of

\title{
Globin gene gains credentials
}

IN a report last year, J. Marks, J.-P. Shaw and C.-K. J. Shen (Nature 321, 785; 1986) added a new member to the $\alpha$-globin gene family. However, it was unclear at the time whether the gene encoded a subunit of a hitherto unknown and unsuspected form of haemoglobin - perhaps one specialized for carrying oxygen at a very early stage of development - or whether, as intimated by $\mathbf{N}$. Proudfoot in a News and Views article (Nature 321,$730 ; 1986$ ) it was merely another pseudogene, one of those ghostly shadows of real genes caught at various stages of decay into randomness. The doubts about the new gene, dubbed a $\theta 1$-globin gene, were stimulated by the unusual nature of the sequence flanking the presumed site of initiation of transcription, where signals regulating the expression of the gene are expected to be found. Although the CCAAT and ATA sequences characteristic of eukaryotic 'promoters' were identified, they were considerably further upstream of the coding sequence of the gene than usual, perhaps, as Proudfoot suggested, too far for the gene to be expressed.

Although direct evidence that the $\theta 1$ globin gene is functional in any species is still lacking, the results reported on pages 717-720 of this issue considerably strengthen the case. Marks's group has now cloned and sequenced the equivalent to the orang-utan $\theta 1$-globin gene from the olive baboon. The availability of the two

states is also in good agreement with the specific heat measured at low temperatures for the metallic glass and the quasicrystal. Similarly, the local moments appearing on the manganese atoms of $\mathrm{Al}_{73} \mathrm{Mn}_{21} \mathrm{Si}_{6}$, deduced from the comparison of low-field and high-field magnetization measurements (R. Bellissuet et al., Léon Brillouin Institute, Saclay), interact magnetically below $5.2 \mathrm{~K}$ in the icosahedral phase with a characteristic spinglass freezing, as previously suggested ${ }^{5}$ by workers at Bell Laboratories in the amorphous state. The magnetism, which disappears in the crystalline $\beta$-phase, could possibly result from a proximity effect of nearest-neighbour manganese atoms.

Heat capacity, transport and optical properties of $\mathrm{Al}-\mathrm{Mn}-(\mathrm{Si})$ were also reported by C. Berger et al. (Lab. d'Etudes des Propriétés Electroniques des Solides, Centre de Recherches sur les Très Basses Températures, Grenoble), J.L. VergerGaugry et al. (Lab. de Thermodynamique et Physico-Chimie Métallurgiques, Grenoble) and J.M. Frigerio et al. (Lab. Optique des Solides, Orsay). Clearly, these properties appear to be strongly influenced by scattering on manganese $d$ resonant states in the aluminium $s p$ conduction band. Whereas the high sequences allows an evolutionary test of whether the $\theta 1$-globin gene is functional in these species. This is because the sequence of a gene can be partitioned into a subset of sites expected to be subject to conservative selective pressure (purifying selection) if the gene is functional, and a subset expected to evolve more rapidly by neutral drift. The expectation is that the difference in rates of evolution of these two subsets should be lost if the gene is a pseudogene. When Marks and colleagues compared the sequences of the orang-utan and olive baboon $\theta 1$-globin genes they found a clear difference in the amount of divergence of the putative selected and non-selected subsets of the sequence: of 27 changes in the putative coding region of the genes, 22 are in the silent third codon positions and only 5 are predicted to alter the sequence of the encoded protein. In fact the third codon positions of this gene seem to be evolving remarkably quickly, so the result is particularly clear-cut. Taken together with the conservation of the unusual promoter organization in the olive baboon $\theta 1$-globin gene, the balance of probability must surely now lie with the gene being functional. If this is indeed so it will be of interest to learn precisely when during development the gene is expressed, and what features the haemoglobin containing the $\theta 1$-subunit may have that suit it to carrying oxygen during that developmental stage.

Geoffrey North

values of the direct current resistivity are of the same order of magnitude as in the amorphous phases, indicating a contribution from structural defects, the frequencydependent conductivity is similar to that in crystalline compounds, presumably with band structure-like effects.

Finally, Pannetier et al. (Centre de Recherches sur les Très Basses Températures, Grenoble) demonstrated the quantization of magnetic flux across a superconducting micronic mesh of aluminium or indium wires reproducing a Penrose tiling, similar to the work ${ }^{6}$ of a group at the University of Pennsylvania. Access to the singular electronic states of such a perfect two-dimensional quasicrystal can soon be expected. But transposition to real alloys is probably more hazardous.

Shechtman, D., Blech, I., Gratias, D. \& Cahn, J.W. Phys. Rev. Lett. 53, 1951 (1984)

. Dubost, B., Lang, J.M., Tanaka, M., Sainfort, P. \& Audier M. Nature 324, 48 (1986).

Lang, J.M., Audier, M., Dubost, B. \& Sainfort, P. J. Cryst. Growth (in the press)

4. Denover, F., Heger, G., Lambert, M. \& Lang, J.M. C.R. Acad. Sc. Seance (in the press)

Hauser, J.J., Chen, H.S. \& Waszczak, J.V. Phys. Rev. B33, 3577 (1986).

6. Behrooz, A. et al. Phys. Rev. Lett. 57, 368 (1986).

P. Guyot is at the Institute National Polytechnique de Grenoble, Domain Universitaire, B.P.75, 384002 St-Martin-D'Hères, Cedex, France. 